\title{
Cinética de degradação e vida-de-prateleira de suco integral de manga
}

\author{
Kinetics of degradation and shelf-life of whole mango juice
}

\section{Anderson do Nascimento Oliveira ${ }^{\mathrm{I}}$ Afonso Mota $\operatorname{Ramos}^{\mathrm{I}}$ José Benício Paes Chaves $^{\mathrm{I}}$ Maria Emília Rodrigues Valente ${ }^{\mathrm{I}}$}

\section{RESUMO}

Este trabalho objetivou estudar a cinética de degradação de suco integral manga Ubá por testes acelerados e estimar sua vida-de-prateleira a $25^{\circ} \mathrm{C}$. Para isso, amostras do produto foram armazenadas em estufas tipo BOD a 25, 35 e $45^{\circ} \mathrm{C}$, providas de iluminação (650lux) 24 horas por dia. As características físico-químicas que mais influenciaram na qualidade do produto foram as coordenadas de cor ( $\left.L^{*} e \Delta E^{*}\right)$ e a concentração de vitamina $C$. A alteração da cor seguiu o modelo cinético de ordem zero, sendo verificado um escurecimento dos produtos mantidos a $35^{\circ} \mathrm{C}$ e $45^{\circ} \mathrm{C}$, enquanto que a degradação da vitamina $C$ foi mais bem explicada pelo modelo de primeira ordem. Houve correlação significativa entre a concentração de vitamina $C$ e os valores de $L^{*}$ e $\Delta E^{*}$ $(P<0,01)$, sugerindo que a degradação dessa vitamina influenciou no escurecimento do produto. Uma vida-deprateleira de 190 dias foi estimada para o suco integral de manga, a $25^{\circ} \mathrm{C}$, utilizando a concentração de vitamina $C$ como parâmetro de qualidade.

Palavras-chave: manga, testes acelerados, vida-de-prateleira, vitamina $C$.

\section{ABSTRACT}

This research aimed to study the kinetic of whole mango juice $c v$. 'Ubá' degradation using accelerated assays and estimate its shelf-life at $25^{\circ} \mathrm{C}$. The products were stored in $B O D$ incubator at $25^{\circ} \mathrm{C}$ (control), $35^{\circ} \mathrm{C}$ and $45^{\circ} \mathrm{C}$ (accelerated conditions), provided with 24 hours a day 650lux lighting. Physico-chemical properties that most influenced the quality of the product were the color coordinates $\left(L^{*}\right.$ and $\left.\Delta E^{*}\right)$ and the vitamin $C$ content. It was found that zero order kinetic model was the best fit to variations in the values of $L^{*}$ and $\Delta E^{*}$, which showed browning of products stored under higher temperatures.
There were significant correlations between changes in vitamin $C$ content and changes in values of $L^{*}$ and $\Delta E^{*}$, suggesting that the degradation of this vitamin contributed to darkening of the product. Shelf-life of 190 days was estimated for whole mango juice stored at $25^{\circ} \mathrm{C}$, using vitamin $C$ content as the limiting quality.

Key words: mango, accelerated assays, shelf-life, vitamin $C$.

\section{INTRODUÇÃO}

A vida-de-prateleira dos alimentos pode ser influenciada por diversos fatores, tais como as condições de processamento, tipo de embalagem, carga microbiana inicial, tempo e temperatura de armazenamento. Dentre estes fatores, a temperatura é geralmente o mais determinante, pois pode acelerar a oxidação de certos nutrientes, e alterar as propriedades nutritivas e sensoriais dos produtos.

Em função das características do alimento, tais como composição nutricional e funcionalidade, vários são os critérios que podem ser utilizados para se determinar o fim da sua vida-de-prateleira. A escolha desse fator limitante depende, no entanto, da natureza das reações deterioradoras que mais limitam a sua qualidade. Ele deve representar adequadamente a degradação do produto, variar de acordo com um modelo bem definido e ser facilmente mensurável (PALAZÓN et al., 2009).

'Programa de Pós-graduação em Ciência e Tecnologia de Alimentos, Departamento de Tecnologia de Alimentos (DTA), Laboratório de Ciência de Frutas e Hortaliças, Universidade Federal de Viçosa (UFV), Campus Universitário, 36570-000, Viçosa, MG, Brasil. E-mail: amramos@ufv.br.*Autor para correspondência. 
Por ser um nutriente presente em grandes concentrações em produtos de origem vegetal e se tratar de um composto termolábil e muito sensível a condições adversas de manipulação, processamento e armazenamento, o nível de degradação de vitamina $\mathrm{C}$ pode ser utilizado como indicador de qualidade e para a estimativa da vida-de-prateleira de sucos de fruta. A oxidação da vitamina $\mathrm{C}$, além de diminuir ou eliminar a atividade vitamínica, gera sabores indesejáveis (ALZUBAIDY \& KHALIL, 2007; TEIXEIRA et al., 2006).

Para alimentos que apresentam vida-deprateleira consideravelmente longa, os testes acelerados de vida-de-prateleira (TAVP) são normalmente empregados. Nestes testes, os produtos são submetidos a condições de armazenamento relativamente severas, bem definidas e controladas, de forma a acelerar as taxas de transformação (MOURA et al., 2007). Finalmente, os dados obtidos nas condições aceleradas são utilizados para estimar a vida-deprateleira do produto nas condições reais de armazenamento (TEIXEIRA NETO et al., 2004).

O suco de manga Ubá é um produto amplamente produzido em escala industrial e consumido em todo Estado de Minas Gerais. No entanto, há uma carência de dados físico-químicos e cinéticos deste produto. Assim, este trabalho objetivou estudar a cinética de degradação do suco integral de manga Ubá, por meio da aplicação do TAVP, e estimar a vida-de-prateleira do produto a $25^{\circ} \mathrm{C}$.

\section{MATERIAIS E MÉTODOS}

O experimento realizado com amostras de suco integral de manga cv. 'Ubá', de uma marca comercial de grande circulação, acondicionadas em garrafas PET de 500mL. As amostras foram adquiridas diretamente da indústria e conduzidas imediatamente ao laboratório para serem armazenadas em estufas, tipo BOD, a $25^{\circ} \mathrm{C}$ (controle), $35^{\circ} \mathrm{C} \mathrm{e} 45^{\circ} \mathrm{C}$ (condições aceleradas), providas de iluminação 24 horas por dia (650lux), de modo a simular as condições reais de armazenamento. $\mathrm{O}$ experimento foi conduzido segundo o delineamento em parcelas subdivididas, com a temperatura de armazenamento inteiramente ao acaso nas parcelas, em duas repetições, e o tempo nas subparcelas.

Os produtos foram analisados quanto a $\mathrm{pH}$, sólidos solúveis totais (SST), acidez titulável (AT), cor instrumental, viscosidade, vitamina C e carotenóides. As análises de $\mathrm{pH}$, SST e AT foram realizadas segundo IAL (2004). As determinações das coordenadas de cor objetiva $\left(\mathrm{L}^{*}, \mathrm{a}^{*} \mathrm{e} \mathrm{b}^{*}\right)$ foram realizadas diretamente em colorímetro Minolta color reader CR-10, sistema CIELAB, previamente calibrado. A diferença total de cor $\left(\Delta E^{*}\right)$ foi calculada de acordo com a equação 1 (LOPES et al., 2005):

$$
\Delta E^{*}=\sqrt{(\Delta L *)^{2}+(\Delta a *)^{2}+\left(\Delta b^{*}\right)^{2}}
$$

Em que $\Delta$ é a variação entre cada coordenada de cor da amostra inicial (tempo zero) e amostra armazenada.

A viscosidade foi determinada em reômetro de cilindros concêntricos tipo Searle, marca Brookfield, modelo R/S plus SST 2000, a $25^{\circ} \mathrm{C}$, utilizando o sensor DG DIN e taxa de deformação de $10 \mathrm{~s}^{-1}$. Adeterminação de vitamina $\mathrm{C}$ foi realizada pelo método oficial da Association of Official Analytical Chemistry (AOAC, 1984). O teor de carotenóides foi determinado por análise espectrofotométrica, conforme metodologia descrita por RODRIGUEZ-AMAYA (2001). Durante o período de armazenamento, os produtos também foram analisados quanto à presença de coliformes termotolerantes, segundo o método 986.33 da AOAC (2002), e Salmonella sp., de acordo com a metodologia descrita por SILVA et al. (2007).

Os dados experimentais foram submetidos à análise de variância (ANOVA) e de regressão em função do tempo, sendo testados os modelos de ordem zero e primeira ordem. A escolha do modelo de melhor ajuste foi realizada a partir da análise da falta de ajuste com os dados experimentais a 5\% de significância e do coeficiente de determinação $\left(\mathrm{R}^{2}\right)$.

Para a degradação da vitamina $\mathrm{C}$, a energia de ativação $\left(E_{a}\right)$ foi determinada graficamente (TEIXEIRA NETO et al., 2004). O fator de aceleração da temperatura (Q10) foi determinado por meio da equação 2, conforme descrito por MOURA et al. (2007). As determinações de Q10 e Ea para as coordenadas de cor $\left(\mathrm{L}^{*} \mathrm{e} \Delta \mathrm{E}^{*}\right.$ ) foram realizadas a partir das equações 3 e 4 , respectivamente, visto que não foram observadas variações significativas $(\mathrm{P}>0,05)$ a $25^{\circ} \mathrm{C}$, (TEIXEIRA NETO et al., 2004).

$$
\begin{aligned}
Q_{10} & =10^{\frac{E_{a}}{0,46 \times T^{2}}} \\
Q_{10} & =\frac{K_{45^{\circ} \mathrm{C}}}{K_{35^{\circ} \mathrm{C}}} \\
E_{a} & =0,46 \times T^{2} \times \log Q_{10}
\end{aligned}
$$

Em que T é a temperatura absoluta e K é a constante de velocidade de degradação.

O efeito simultâneo da temperatura e do tempo de armazenamento foi analisado por metodologia de superfície de respostas, conforme descrito por ARRUDA (2003). Para a determinação da vida-deprateleira do produto, utilizou-se a concentração de 
vitamina $\mathrm{C}$ como limitante da qualidade (TEIXEIRA et al., 2006; AL-ZUBAIDY \& KHALIL, 2007), considerando o nível máximo aceitável a concentração equivalente a $50 \%$ do teor inicial no produto. As análises estatísticas foram realizadas utilizando-se o programa estatístico SAS (Statistical Analysis System - SAS Institute Inc., Cary, NC, USA), versão 9.1.

\section{RESULTADOS E DISCUSSÃO}

Observou-se que para os parâmetros teor de carotenóides, SST, pH e viscosidade não foram verificados efeitos significativos de interação tempotemperatura de armazenamento $(\mathrm{P}>0,05)$, assim como destes fatores isoladamente. Os valores de SST e pH mostraram-se de acordo com os valores estabelecidos pela legislação (BRASIL, 2000), com valores médios de $3,72 \mathrm{e} 15,5^{\circ} \mathrm{Brix}$, respectivamente. Resultado semelhante foi verificado por FARAONI (2006), que não encontrou variação significativa $(\mathrm{P}>0,05)$ para SST em polpa de manga Ubá armazenada sob refrigeração durante seis meses e em diferentes embalagens. Os baixos valores de $\mathrm{pH}$ revelam a boa estabilidade microbiológica do produto durante todo o período estudado. De fato, não foram observadas as presenças de coliformes termotolerantes e Salmonella sp. durante o armazenamento, estando em conformidade com legislação (BRASIL, 2001).

A viscosidade dos sucos, a uma taxa de deformação de $10 \mathrm{~s}^{-1}$, não variou significativamente ( $\mathrm{P}>0,05)$ ao longo do tempo para todas as temperaturas de armazenamento, com valor médio de 503,75mPa.s. Resultados semelhantes foram obtidos por PELEGRINE et al. (2000), para polpa de manga Keitt (425mPa.s a $\left.50 \mathrm{~s}^{-1} \mathrm{e} 30^{\circ} \mathrm{C}\right)$.

A concentração de carotenóides nos sucos armazenados nas diferentes temperaturas também não variou significativamente $(\mathrm{P}>0,05)$ durante a estocagem, apresentando um valor médio de $15,67 \mu \mathrm{g} \mathrm{g}^{-1}$. Resultados semelhantes foram obtidos por GODOY \& RODRIGUEZ-AMAYA (1987). Esses autores verificaram uma concentração de $15,74 \mu \mathrm{g} \mathrm{g}^{-1}$ em polpa de manga Golden e após 10 meses de armazenamento em garrafas de vidro, a temperatura ambiente, a concentração foi de $13,13 \mu \mathrm{g} \mathrm{g}^{-1}$. Com 14 meses de armazenamento, a concentração diminuiu para 7,51 $\mathrm{g} \mathrm{g} \mathrm{g}^{-1}$. Portanto, é possível que o tempo de armazenamento do suco de manga neste estudo não tenha sido suficiente para haver perdas significativas na concentração de carotenóides, mesmo nas condições aceleradas. As concentrações de carotenóides no suco integral de manga estão de acordo com os resultados encontrados por RIBEIRO et al. (2007), para polpa de manga Ubá $\left(22,20 \mu \mathrm{g} \mathrm{g}^{-1}\right)$.

Com relação à cor, observou-se redução significativa da luminosidade $(\mathrm{P}<0,05)$ para os sucos armazenados nas temperaturas de 35 e $45^{\circ} \mathrm{C}$. Observouse que o modelo cinético de ordem zero apresentou melhor ajuste aos dados experimentais, com constantes cinéticas de $0,0187 \operatorname{dias}^{-1}\left(35^{\circ} \mathrm{C}\right)$ e $0,0487 \operatorname{dias}^{-1}\left(45^{\circ} \mathrm{C}\right)$. Semelhantemente, a diferença total de cor $\left(\Delta \mathrm{E}^{*}\right)$ também variou significativamente $(\mathrm{P}<0,05)$ para os sucos armazenados a 35 e $45^{\circ} \mathrm{C}$, com constantes cinéticas de $0,0313 \operatorname{dias}^{-1}\left(35^{\circ} \mathrm{C}\right)$ e $0,110 \operatorname{dias}^{-1}\left(45^{\circ} \mathrm{C}\right)$, para o modelo de ordem zero, de melhor ajuste. Estas variações indicam alteração na coloração ao longo do tempo, possivelmente resultante de reações de escurecimento não enzimático, como a oxidação da vitamina $C$ e reação de Maillard, que ocasionam a formação de pigmentos escuros no produto, aceleradas pelas temperaturas mais elevadas (AL-ZUBAIDY \& KHALIL, 2007; TEIXEIRA et al., 2006).

Para a degradação da vitamina $\mathrm{C}$, o modelo de primeira ordem (Figura 1) mostrou-se mais adequado para representar a degradação com o tempo. A concentração inicial de vitamina $\mathrm{C}$ no suco foi de $26,8 \mathrm{mg} 100 \mathrm{~mL}^{-1}$, a qual reduziu significativamente $(\mathrm{P}<0,05)$ para os produtos armazenados em todas as temperaturas. As constantes cinéticas de degradação foram de $0,0039 \mathrm{dias}^{-1}\left(25^{\circ} \mathrm{C}\right), 0,0080 \mathrm{dias}^{-1}\left(35^{\circ} \mathrm{C}\right) \mathrm{e}$ $0,0161 \mathrm{dias}^{-1}\left(45^{\circ} \mathrm{C}\right)$, demonstrando a sensibilidade da vitamina $\mathrm{C}$ à temperatura de armazenamento, conforme observado por TEIXEIRA et al. (2006), para goiabas industrializadas e por AL-ZUBAIDY \& KHALIL (2007), para suco de limão. As concentrações encontradas neste trabalho foram superiores às determinadas por BERNIZ (1984) em polpas de manga Ubá, que verificou concentrações de 15,7 a $17,4 \mathrm{mg} 100 \mathrm{~mL}^{-1}$ com 10 a $16 \%$ de sólidos solúveis totais. No entanto, concentrações maiores de vitamina $\mathrm{C}$ em polpas de manga Ubá foram observadas por FARAONI (2006), para polpas pasteurizadas por diferentes binômios tempo-

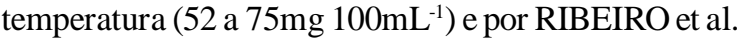
(2007), 77,71mg 100 $\mathrm{g}^{-1}$.

Com relação ao efeito da temperatura, observou-se que a degradação da vitamina $\mathrm{C}$ seguiu o modelo de Arrhenius $\left(\mathrm{R}^{2}=0,998\right)$ (Figura 2). Os parâmetros cinéticos Q10 e Ea obtidos para luminosidade $\left(\mathrm{L}^{*}\right), \Delta \mathrm{E}^{*}$ e vitamina $\mathrm{C}$ foram, respectivamente, 2,60 e $17,00 \mathrm{kcal} \mathrm{mol}^{-1}\left(\mathrm{~L}^{*}\right), 3,51 \mathrm{e}$ 22,30kcal mol-1 $\left(\Delta \mathrm{E}^{*}\right)$ e 2,13 e 13,41 kcal mol ${ }^{-1}$ (vitamina C). Estes resultados indicam que a cor do suco integral 


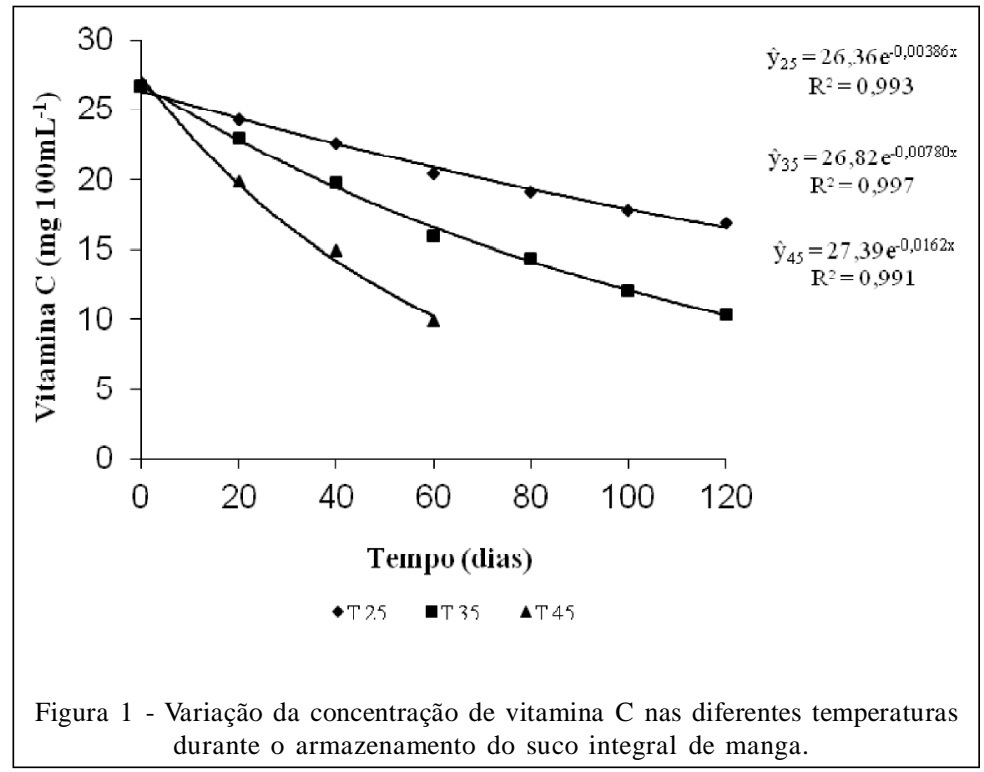

de manga é mais dependente da temperatura que a concentração de vitamina C. Os valores de Ea para a cor são compatíveis com as alterações na cor em produtos alimentícios, que de acordo com LUND (1979), deve estar na faixa de 10-30 $\mathrm{kcal} \mathrm{mol}^{-1}$. Para a vitamina $\mathrm{C}$, os resultados encontrados estão de acordo os encontrados na literatura. Por exemplo, ALZUBAIDY \& KHALIL (2007) verificaram que para suco de limão nas temperaturas de 25,35 e $45^{\circ} \mathrm{C}$ e em diferentes concentrações $\left(9^{\circ} \mathrm{Brix}\right.$ e $\left.50^{\circ} \mathrm{Brix}\right)$, foram obtidos valores de 13,8 e $15,3 \mathrm{kcal} \mathrm{mol}^{-1} \mathrm{e} \mathrm{Q}_{10\left(25-35^{\circ} \mathrm{C}\right)}$ de 1,486 e 1,489 , respectivamente. Resultados semelhantes também foram obtidos por TORALLES et al. (2008), para purê de pêssego na faixa de $70-90^{\circ} \mathrm{C}\left(\mathrm{E}_{\mathrm{a}} \mathrm{de}\right.$ $10,76 \mathrm{kcal} \mathrm{mol}^{-1} \mathrm{e} \mathrm{Q}_{10}$ de 1,45 a 1,61 ).

Os modelos matemáticos obtidos a partir da análise de regressão múltipla para a degradação de vitamina $C, L^{*} \mathrm{e} \Delta \mathrm{E}^{*}$ são mostrados nas equações 5-7: Vitamina $\mathrm{C}\left(\mathrm{mg} 100 \mathrm{~mL}^{-1}\right)=26,89+0,000 \mathrm{t}^{2}-$ $0,0067 \mathrm{tT}\left(\mathrm{R}^{2}=0,990\right)$

$$
\Delta \mathrm{E}^{*}=9,59-0,617 \mathrm{~T}-0,00043 \mathrm{t}^{2}+0,0088 \mathrm{~T}^{2}+
$$

$0,0028 \mathrm{tT}\left(\mathrm{R}^{2}=0,937\right)$

$$
\mathrm{L}^{*}=50,27-0,00083 \mathrm{~T}^{2}-0,00053 \mathrm{tT}\left(\mathrm{R}^{2}=\right.
$$

$0,848)$

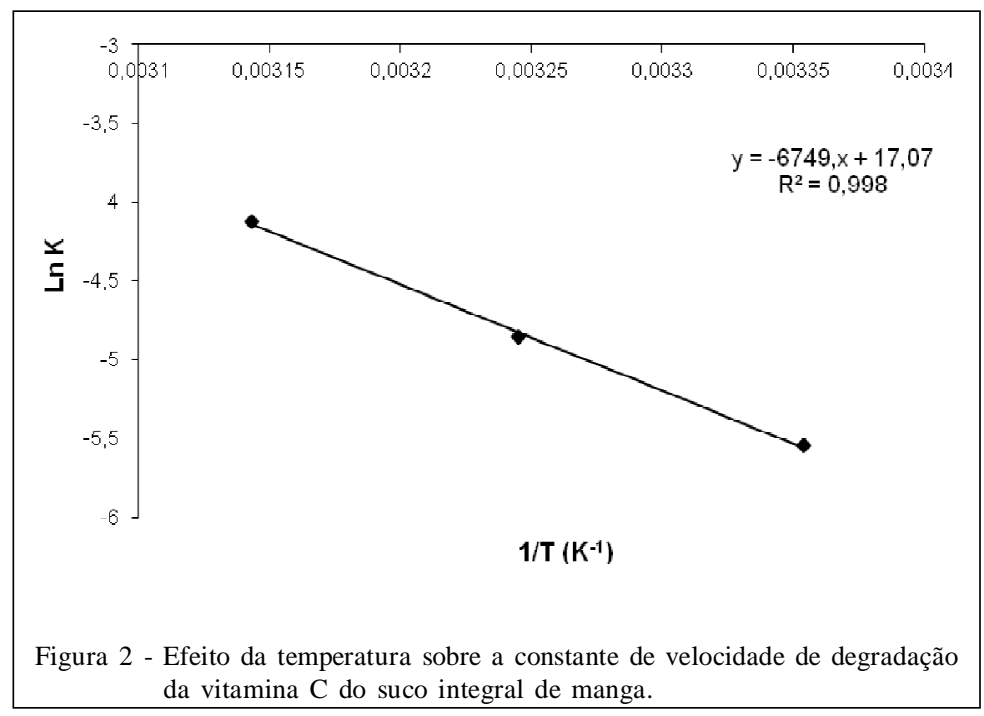

Ciência Rural, v.43, n.1, jan, 2013. 
Em que t e $\mathrm{T}$ são o tempo (dias) e a temperatura de armazenamento $\left({ }^{\circ} \mathrm{C}\right)$, respectivamente.

Todos os modelos ajustados foram significativos ao nível de $0,1 \%$ de probabilidade.

Os gráficos de superfície de respostas e de contornos para a degradação da vitamina $\mathrm{C}$ e de $\Delta \mathrm{E}^{*}$ estão apresentados na figura 3 . A análise dos gráficos da figura 3 permite observar que a degradação da vitamina $\mathrm{C}$ está possivelmente relacionada com as alterações na cor, o que se deve a formação de pigmentos escuros (melanoidinas), resultantes das reações de oxidação (AL-ZUBAIDY \& KHALIL, 2007; TEIXEIRA et al., 2006). De fato, por meio da análise estatística, observou-se correlação significativa (correlação de Pearson) entre a degradação da vitamina $\mathrm{C}$ e as coordenadas de cor $\mathrm{L}^{*}(0,91) \mathrm{e} \Delta \mathrm{E}^{*}(-0,88)$, em nível de $0,1 \%$ de probabilidade. Ou seja, a degradação da vitamina $\mathrm{C}$ está relacionada com a diminuição dos valores de $\mathrm{L}^{*}$ (escurecimento) e aumento dos valores $\operatorname{de} \Delta \mathrm{E}^{*}$ (alteração da cor característica).

A vida-de-prateleira do suco integral de manga Ubá a $25^{\circ} \mathrm{C}$ foi estimada considerando a concentração mínima aceitável de vitamina $\mathrm{C}$ equivalente a $50 \%$ da concentração inicial (tempo zero). Com base neste critério, a vida-de-prateleira determinada para o produto armazenado na temperatura de $35^{\circ} \mathrm{C}$ foi de 89 dias. Considerando também o valor de $\mathrm{Q}_{10}$ calculado para a vitamina $\mathrm{C}(2,13)$, foi possível estimar a vida-de-prateleira do suco a $25^{\circ} \mathrm{C} \mathrm{em} 190$ dias, equivalente a aproximadamente seis meses e meio de armazenamento. Este valor encontra-se próximo ao declarado pelo fabricante, que é de oito meses.

\section{CONCLUSÃO}

As alterações nas coordenadas de cor ( $\left.\mathrm{L}^{*} \mathrm{e} \Delta \mathrm{E}^{*}\right)$ e na concentração de vitamina $C$ foram as que mais afetaram a qualidade do suco de manga durante o armazenamento.

$\mathrm{O}$ teste acelerado de vida-de-prateleira permitiu estimar em 190 dias a vida-de-prateleira do suco integral de manga a $25^{\circ} \mathrm{C}$, utilizando a concentração de vitamina $\mathrm{C}$ como parâmetro, mostrando ser uma ferramenta bastante útil às indústrias, quando não for viável a realização de estudos nas condições normais de armazenamento.

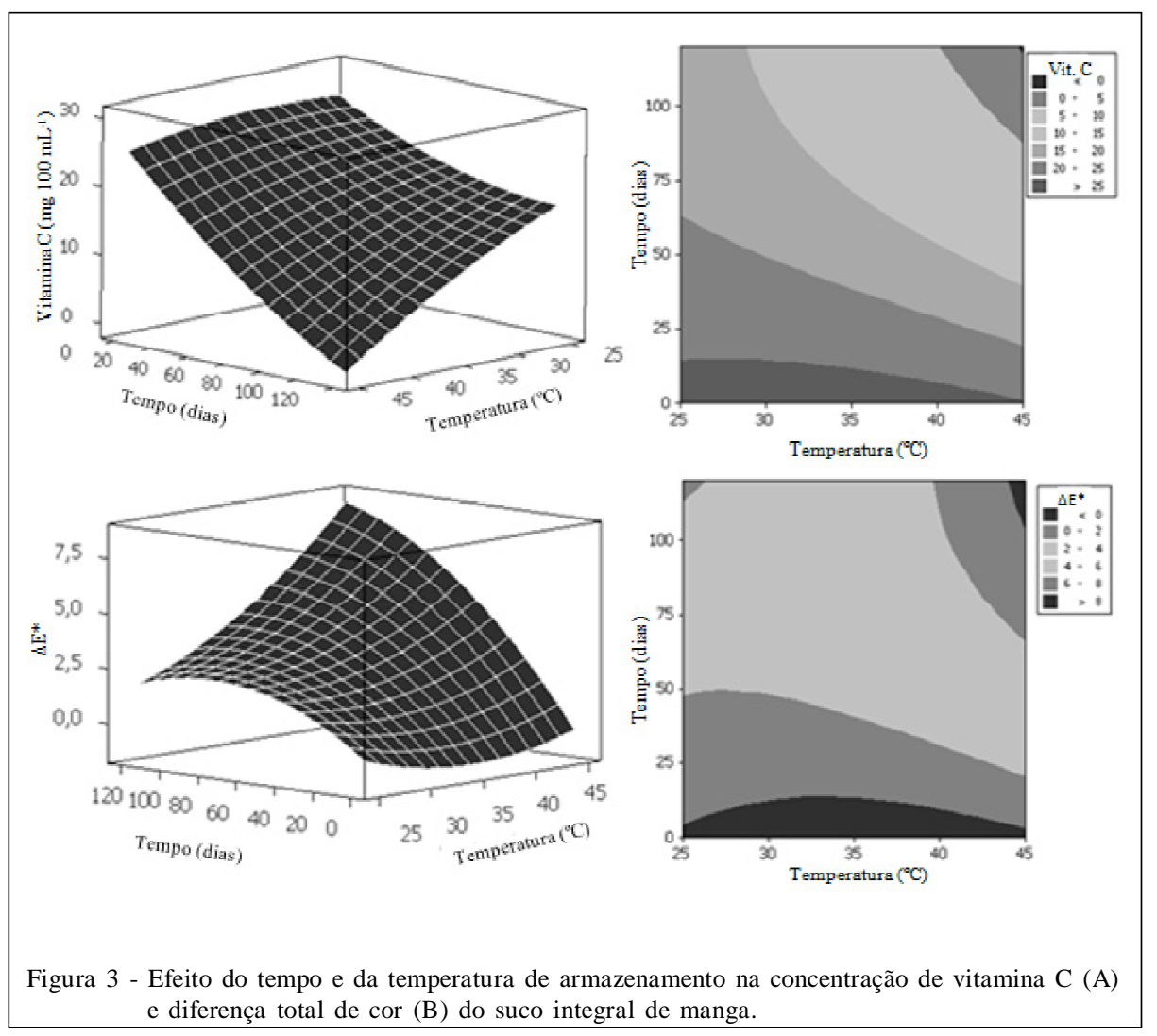

Ciência Rural, v.43, n.1, jan, 2013. 


\section{AGRADECIMENTOS}

Os autores agradecem à Fundação de Amparo à Pesquisa do estado de Minas Gerais (FAPEMIG) e ao Conselho Nacional de Desenvolvimento Científico e Tecnológico (CNPq) pelo auxílio financeiro.

\section{REFERÊNCIAS}

AL-ZUBAIDY, M.M.I.; KHALIL, R.A. Kinetic and prediction studies of ascorbic acid degradation in normal and concentrate local lemon juice during storage. Food Chemistry, v.101, n.1, p.254-259, 2007. Disponível em <http://www.sciencedirect.com/ science/article/pii/S0308814606000811>. Acesso em: $12 \mathrm{dez}$. 2010. doi: 10.1016/j.foodchem.2006.01.024.

ASSOCIATION OF OFFICIAL ANALITICAL CHEMISTS (AOAC). Official methods of analysis of the of Official Analytical Chemists. 14.ed. Washington, D.C., 1984. 1141p.

ASSOCIATION OF OFFICIAL ANALYTICAL CHEMISTS (AOAC). Official methods of analysis of the Association of Official Analytical Chemists. 17.ed. Washington, 2002. 570p.

ARRUDA, A.F.P. Estudo da estabilidade de néctar de manga (Mangifera indica L.) envasado em garrafas PET, comparado com envasados em embalagem cartonada $\mathrm{e}$ lata de alumínio. 2003. 112f. Dissertação (Mestrado em Tecnologia de Alimentos) - Faculdade de Engenharia de Alimentos, Universidade Estadual de Campinas, SP.

BERNIZ, P.J. Avaliação industrial de variedades de manga (Mangifera indica L.) para elaboração de néctar. 1984. 57F. Dissertação (Mestrado em Ciência e Tecnologia de Alimentos) - Universidade Federal de Viçosa, MG.

BRASIL. Agência Nacional de Vigilância Sanitária (ANVISA) Ministério da Saúde. Resolução - RDC n.12, de 2 de janeiro de 2001. Diário Oficial da República Federativa do Brasil. Aprova o Regulamento Técnico sobre Padrões Microbiológicos para Alimentos. Disponível em: <http://www.anvisa.gov.br/legis/ resol/12_01rdc.htm>. Acesso em: 23 maio, 2011.

BRASIL. Ministério da Agricultura e do Abastecimento. Instrução Normativa n.1, de 7 de janeiro de 2000. Diário Oficial da República Federativa do Brasil. Aprova o Regulamento Técnico Geral para Fixação dos Padrões de Identidade e Qualidade para Polpa de Frutas. Disponível em: $<$ http://extranet.agricultura.gov.br/sislegis-consulta/ consultarLegislacao.do?operacao $=$ visualizar $\& i d=7777>$. Acesso em: 23 maio, 2011.

FARAONI, A.S. Efeito do tratamento térmico, do congelamento e da embalagem sobre o armazenamento da polpa de manga orgânica (Mangifera indica L) cv. 'Ubá'. 2006. 117f. Dissertação (Mestrado em Ciência e Tecnologia de Alimentos) - Departamento de Tecnologia de Alimentos, Universidade Federal de Viçosa, MG.

GODOY, H.T.; RODRIGUES-AMAYA, D.B. Changes in individual carotenoids on processing and storage of mango (Mangifera indica) slices and puree. International Journal of Food Science Technology, v.22, n.5, p.451-460, 1987.
Disponível em: <http://onlinelibrary.wiley.com/doi/10.1111/ j.1365-2621.1987.tb00510.x/abstract $>$. Acesso em: 15 jan. 2011. doi: 10.1111/j.1365-2621.1987.tb00510.x.

INSTITUTO ADOLFO LUTZ. Normas analíticas do Instituto Adolfo Lutz: métodos químicos e físicos para análises de alimentos. 4.ed. São Paulo, 2004. 1004p.

LUND, D.B. Effect of commercial processing on nutrients. Food Technology, v.33, n.2, p.28-35, 1979.

MOURA, S.C.S.R. et al. Determinação da vida-de-prateleira de maçã-passa por testes acelerados. Ciência e Tecnologia de Alimentos, v.27, n.1, p.141-148, 2007. Disponível em: < http:/ /www.scielo.br/pdf/cta/v27n1/24.pdf>. Acesso em: 22 mar. 2011. doi: 10.1590/S0101-20612007000100025.

PALAZÓN, M.A. et al. Determination of shelf-life of homogenized apple-based beikost storage at different temperatures using Weibull hazard model. Food Science and Technology, v.42, n.1, p.319-326, 2009. Disponível em: <http://www.sciencedirect.com/science/article/pii/ S0023643808000832>. Acesso em: 24 jun. 2012. doi: 10.1016/ j.lwt.2008.03.011.

PELEGRINE, D.H. et al. Estudo da viscosidade aparente das polpas de manga (Keitt) e abacaxi (Pérola). Ciência e Tecnologia de Alimentos, v.20, n.1, p.128-131, 2000. Disponível em: <http:/ /www.scielo.br/scielo.php?script=sci_arttext\&pid=S0101$20612000000100024 \& \operatorname{lng}=\mathrm{en} \& \mathrm{nrm}=\mathrm{iso} \& \operatorname{lng}=\mathrm{pt}>$. Acesso em: 25 maio, 2011. doi: 10.1590/S0101-20612000000100024.

RIBEIRO, S.M.R. et al. Antioxidant in Mango (Mangifera indica L.) Pulp. Plant Foods for Human Nutrition, v.62, n.1, p.1317, 2007. Disponível em: <http://www.springerlink.com/content/ d3107314mm0uv5n7/fulltext.pdf $>$. Acesso em: 25 maio, 2011. doi: 10.1007/s11130-006-0035-3.

RODRIGUEZ-AMAYA, D.A. Guide to carotenoids analysis in food. Washington: International Life Sciences Institute, 2001. 64p.

SILVA, N. et al. Manual de métodos de análise microbiológica de alimentos. 3.ed. São Paulo: Livraria Varela, 2007. 535p.

TEIXEIRA, J. et al. Degradação do ácido ascórbico em goiabada industrializada submetida a diferentes condições de estocagem. Alimentos e Nutrição, v.17, n.3, p.281-286, 2006. Disponível em: <http://serv-bib.fcfar.unesp.br/seer/index.php/ alimentos/article/view/275/267>. Acesso em: 13 fev. 2011.

TEIXEIRA NETO, R.O. et al. Introdução à cinética de reação em alimentos. In: MOURA, S.C.S.R. Reações de transformação e vida-de-prateleira de alimentos processados. Campinas: ITAL, 2004. Cap.3, p.63-83.

TORALLES, R.P. et al. Determinação das constantes cinéticas de degradação do ácido ascórbico em purê de pêssego: efeito da temperatura e concentração. Ciência e Tecnologia de Alimentos, v.28, n.1, p.18-23, 2008. Disponível em: <http:/ /www.scielo.br/pdf/cta/v28n1/03.pdf>. Acesso em: 20 abr. 2011. doi: 10.1590/S0101-20612008000100004. 